\title{
Pivotal role of anterior cingulate cortex in working memory after traumatic brain injury in youth
}

\author{
Fabienne Cazalis ${ }^{1,2 *}$, Talin Babikian', Christopher Giza ${ }^{3}$, Sarah Copeland ${ }^{3}$, David Hovda ${ }^{3}$ and Robert F. Asarnow' \\ 1 Psychiatry and Biobehavioral Sciences, University of California Los Angeles David Geffen School of Medicine, Los Angeles, CA, USA \\ 2 Department of Anatomy, Ross University School of Medicine, Roseau, Commonwealth of Dominica \\ ${ }^{3}$ Department of Neurosurgery, University of California Los Angeles David Geffen School of Medicine, Los Angeles, CA, USA
}

\section{Edited by:}

Marten Risling, Karolinska Institutet,

Sweden

Reviewed by:

Hans Lindå, Karolinska Institutet,

Sweden

Frank Krueger, George Mason

University, USA

${ }^{*}$ Correspondence:

Fabienne Cazalis,

Department of Anatomy,

Ross University School of Medicine,

P.O. Box 266, Roseau, Commonwealth

of Dominica.

e-mail:fcazalis@rossu.edu
In this fMRI study, the functions of the anterior cingulate cortex (ACC) were studied in a group of adolescents who had sustained a moderate to severe traumatic brain injury (TBI). A spatial working memory task with varying working memory loads, representing experimental conditions of increasing difficulty, was administered. In a cross-sectional comparison between the patients and a matched control group, patients performed worse than Controls, showing longer reaction times and lower response accuracy on the spatial working memory task. Brain imaging findings suggest a possible double-dissociation: activity of the ACC in the TBI group, but not in the Control group, was associated with task difficulty; conversely, activity of the left sensorimotor cortex (ISMC) in the Control group, but not in the TBI group, was correlated with task difficulty. In addition to the main cross-sectional study, a longitudinal study of a group of adolescent patients with moderate to severe TBI was done using fMRI and the same spatial working memory task. The patient group was studied at two time-points: one time-point during the post-acute phase and one time-point 12 months later, during the chronic phase. Results indicated that patients' behavioral performance improved over time, suggesting cognitive recovery. Brain imaging findings suggest that, over this 12-month period, patients recruited less of the ACC and more of the ISMC in response to increasing task difficulty. The role of ACC in executive functions following a moderate to severe brain injury in adolescence is discussed within the context of conflicting models of the ACC functions in the existing literature.

Keywords: traumatic brain injury, diffuse axonal injury, functional MRI, working memory, anterior cingulate cortex, executive functions, adolescent

\section{INTRODUCTION}

Anterior cingulate cortex (ACC) activity is reportedly related to task difficulty, in healthy subjects (Botvinick et al., 2004) as well as in clinical populations, such as patients with post-traumatic stress disorder (Shaw et al., 2009) or patients with traumatic brain injury (TBI) (Rasmussen et al., 2008; Kohl et al., 2009). The exact role of the ACC in executive functions is still debated, with most prominent theories suggesting a role in cognitive control, including error detection, conflict monitoring, and/or task switching (Botvinick et al., 2004; Kerns et al., 2004; Ullsperger and Von Cramon, 2004; Carter and van Veen, 2007; Hyafil et al., 2009).

Executive function is affected by TBI, independently of the presence/absence of cortical lesions (Metting et al., 2007; SanchezCarrion et al., 2008). It has been suggested that it is diffuse axonal injury - commonly observed after TBI - that disrupts integrative networks and that is the main cause of executive functions impairment following TBI (Ghajar and Ivry, 2008; Niogi et al., 2008). This makes TBI a very interesting neuropsychological model for studying the role of ACC in executive functions.

In this study, we investigated ACC activation in relation to task difficulty, using a working memory task in adolescent patients with moderate to severe TBI. Our hypotheses were consistent with the literature: we expected that patients in the cross-sectional study would present lower performance than controls, notably with longer reaction times, and an alteration of their pattern of cerebral activation as compared to controls. We also expected patients in the longitudinal study to exhibit improvement of performance at Time 2 as compared to Time 1, as well as a change in brain imaging.

\section{MATERIALS AND METHODS}

This fMRI study of working memory in adolescents with TBI was conducted in two stages. First, a cross-sectional design compared youth with TBI to matched Controls. Secondly, a longitudinal study was conducted on a subset of the adolescent TBI patients who were reevaluated 12 months after their initial exam.

The spatial working memory task was parametrically designed in order to allow study of the effect of working memory load (WML) on brain activation.

\section{PATIENTS}

Participants included in this study presented with the following characteristics: 13-18 years of age; normal vision (contact lenses were accepted); right handedness; English skills sufficient enough to understand instructions; no previous history of major neurological, developmental, or psychiatric disorders (as stated by the parents of the patients during the inform consent process); and safety compliance to MRI. Recruitment and consent processes were approved by the UCLA Institutional Review Board (IRB). 
Traumatic brain injury patients were recruited from two Pediatric ICUs within Los Angeles County (UCLA Medical Center and Harbor-UCLA Medical Center) as well as a local rehabilitation facility. Patients presented with moderate to severe non-penetrating TBI. Glasgow Coma Scale score (at intake at Emergency Department or post-resuscitation) was between 3 and 12, although three patients with GCS > 12 were included, since they presented with abnormalities on clinical imaging and therefore were classified as having moderate TBI. Severe TBI was defined by a GCS of 8 or less. All TBI patients exhibited diffuse axonal injury, as identified on FLAIR and T2 star MRI sequences. Tables 1 and 2 describe the study groups.

\section{PARADIGM DESCRIPTION}

During the functional MRI exam, subjects performed a parametric cognitive task in an event-related design. They received instructions for the task prior to the exam. Participants responded by pressing a four-button keyboard that was placed under the subject's right hand. Responses and response times (RTs) were recorded.

The task was an original non-verbal spatial working memory task that required participants to reproduce visual sequences (Figure 1). A set of four positions was presented on the screen, as shown in Figure 1. Photographs of fruits and vegetables appeared sequentially, forming a spatio-temporal sequence that subjects were instructed to reproduce using the keyboard. Each picture was sufficiently detailed to be identifiable but sufficiently complex to prevent verbal description (Pepin Press, 2006a,b). The use of non-verbal material was sine qua non to avoid bilingualism being a confound, since exclusion of bilingual children was not a reasonable option (more than half of the children of Los Angeles, California, are bilingual, according to http://www.mla.org). The task was programmed using Matlab (The MathWorks Inc.).

Table 1 | Description of TBI and Control groups for the crosssectional study.

\begin{tabular}{lll}
\hline & TBI & Controls \\
\hline Number of subjects (males/females) & $11(9 / 2)$ & $12(4 / 8)$ \\
Mean age (years) \pm SD (range) & $16.0 \pm 1.5$ & $15.4 \pm 1.5$ \\
Mean delay post-injury & $(14-18)$ & $(13-18)$ \\
(months) \pm SD (range) & $7.0 \pm 2(3-17)$ & \\
Mean Glasgow coma scale & &
\end{tabular}

score \pm SD (range)

Table 2 | Description of TBI group for the longitudinal study.

\begin{tabular}{ll}
\hline & LongitudinalTBI \\
\hline Number of subjects (males/females) & $6(5 / 1)$ \\
Mean age (years) \pm SD (range) at Time 1 & $16.7 \pm 0.7(15.8-17.4)$ \\
Mean age (years) \pm SD (range) at Time 2 & $17.7 \pm 0.8(16.7-18.5)$ \\
Mean delay post-injury (months) \pm SD & $4.5 \pm 1.0$ (3-6) \\
(range) at Time 1 & \\
Mean delay post-injury (months) \pm SD & $16.5 \pm 3.1(14-22)$ \\
(range) atTime 2 & \\
Mean Glasgow coma scale score \pm SD (range) & $9.4 \pm 5.1(3-14)$
\end{tabular}

The task included four conditions that were defined by the number of items to be manipulated in working memory, i.e., WML: Baseline (1 item); Low WML (3 items); Intermediate WML (4 items); High WML ( 5 items). There were 30 trials per condition. Conditions were alternated pseudo-randomly.

\section{MRI EXAM}

The MRI exam was conducted on a 3-T scanner (Siemens, Allegra) and included the following sequences:

- Structural T2 $(\mathrm{TE}=56 ; \mathrm{TR}=4040 \mathrm{~ms} ; \mathrm{FOV}=200 \mathrm{~mm}$; voxel size $=0.8 \mathrm{~mm} \times 0.8 \mathrm{~mm} \times 5.0 \mathrm{~mm} ; 25$ slices of $5 \mathrm{~mm}$; distance of $1.3 \mathrm{~mm}$; axial orientation; order of acquisition = interleaved)

- EPI BOLD $(\mathrm{TE}=30 \mathrm{~ms}$; TR $=2000 \mathrm{~ms}$; FOV = $200 \mathrm{~mm}$; voxel size $=3.125 \mathrm{~mm} \times 3.125 \mathrm{~mm} \times 3 \mathrm{~mm}$; 33 slices; axial orientation; order of acquisition = interleaved; 500 volumes acquired)

- $\mathrm{T}^{*} 3 \mathrm{D}$ flash (48 slices, voxel size $=1 \mathrm{~mm} \times 0.5 \mathrm{~mm} \times 2 \mathrm{~mm}$; $\mathrm{FOV}=256 \mathrm{~mm} ; \mathrm{TR}=57 \mathrm{~ms} ; \mathrm{TE}=20 \mathrm{~ms}$; flip angle $=20^{\circ}$ )

- A 3D volume structural sequence was acquired on a 1.5-T scanner (Siemens, Sonata) on the same day as functional scanning: MP-RAGE sequence (180 sagittal slices; FOV $=256 \mathrm{~mm}$, voxel size $=1 \mathrm{~mm} \times 1 \mathrm{~mm} \times 1 \mathrm{~mm}$; TR $=1900 \mathrm{~ms} ; \mathrm{TE}=4.4 \mathrm{~ms}$; $\mathrm{TI}=1100 \mathrm{~ms}$; flip angle $=15^{\circ}$ )

\section{BEHAVIORAL DATA PROCESSING}

Two behavioral measures were used for statistical analyses: response accuracy and RT. Performance on both of these measures was recorded for each of the four conditions, representing WML [1-3-4-5]. Two groups were compared in the cross-sectional study: TBI and Control groups. For the Control group, behavioral

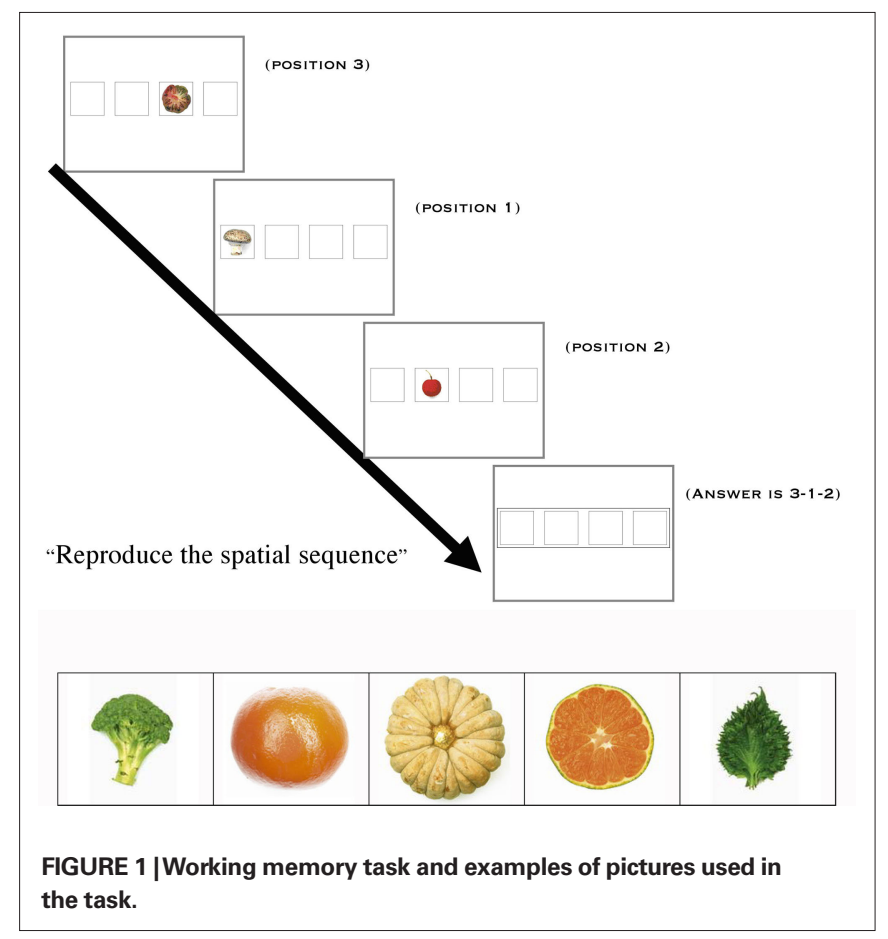


data was available for 11 subjects. A subset of the TBI subjects, for whom data was available at two time-points, was included in the longitudinal study.

\section{Cross-sectional study}

Non-parametric Spearman correlations (two-tailed) were used to determine associations between the behavioral measures of response accuracy and RT with WML. A linear mixed model with post hoc (LSD) analyses were used to characterize case/control differences, with the between subject factor of Group (TBI or Control) and the within-subject factor of WML [1-3-4-5].

\section{Longitudinal study}

Because of the small sample size of the subgroup of the subjects for whom longitudinal data was acquired, simple paired $t$-tests were used for within-group comparison between Time 1 and Time 2 .

\section{MRI DATA PROCESSING}

Functional MRI data were preprocessed and processed using the FSL package (Smith et al., 2004; Woolrich et al., 2009).

\section{Preprocessing}

Preprocessing included motion correction, brain extraction, spatial smoothing (FWHM $=5 \mathrm{~mm}$ ), and high pass temporal filtering (66s).

\section{Processing: first-level analysis}

Data were processed at the individual level according to their event-related design, for a total of five events-lists. Correct trials constituted the first four events, corresponding to the number of items loaded in working memory (WML): WML = 1 ("Baseline"); $\mathrm{WML}=3$ ("Low WML"); WML = 4 ("Intermediate WML"); $\mathrm{WML}=5$ ("High WML"). Failed and missed trials were assembled together in a fifth event that was excluded from analysis. Whitening was appended to data estimation, after convolution with double Gamma hemodynamic response function. Temporal derivative was applied, as well as temporal filtering. Four contrasts were used for this study:

- “Low WML” (Low WML versus Baseline)

- "Intermediate WML" (Intermediate WML versus Baseline)

- "High WML" (High WML versus Baseline)

- "WML increase" (High WML versus Low WML)

Individual activation maps were registered on the standard MNI space through the following steps: structural T2 was the initial image (linear - normal search - 7 DOF), 3D structural volume was the main structural image (linear - normal search - 12 DOF) and the MNI template ("avg152T1_brain" in FSL) was the standard space (linear - normal search - 12 DOF).

\section{Processing: higher-level analysis}

Higher-level analyses were processed at the group level (TBI and Control), using a fixed effects model. The measures for the crosssectional study were: "mean Control group" and "mean TBI group" for the above-mentioned four contrasts. The contrasts for the longitudinal study were "mean TBI group Time 1" and "mean TBI group Time 2." In addition to a voxel threshold of $p=0.05$, all maps were thresholded at the cluster level $(Z=1.8)$ in order to correct for multiple comparison. The template space was used as background for rendering.

\section{Qualitative analysis}

A complete list of all activated areas was compiled, using anatomical atlases (Mai et al., 2004; Ratiu and Talos, 2006) for correct determination of areas.

\section{Quantitative analysis: regions of interest}

Our choice for the regions of interest (ROI), determined by our working hypothesis, included the ACC. In an ideally large study, we would have compared the ACC activity with all the areas reportedly involved in spatial working memory processing (Wager and Smith, 2003; D'Esposito, 2008). However, because of the sample size of our study, we focused on the comparison of ACC with the left sensorimotor cortex (ISMC) because involvement of this later area has been well demonstrated in spatial working memory studies (Shaw et al., 2009) as well as in studies of mental imagery (Fiehler et al., 2008; Hanakawa et al., 2008; Sack et al., 2008).

We defined two spherical ROI according to their MNI coordinates and radius length $(\mathrm{mm})$ :

- Left sensorimotor cortex: $x=-40 ; y=-32 ; z=56 ; r=20$

- Anterior cingulate cortex : $x=0 ; y=34 ; z=28 ; r=14$

Regions of interests were used as masks in order to retrieve individual time-series values within these areas, using the "avwmeants" function included in the FSL package, hence obtaining the intensity of the betas based on the GLM model.

Statistical analyses were conducted for each ROI:

- Within-subjects differences between contrasts were explored using repeated measures ANOVAs. One-tailed $t$-tests were used for post hoc analyses.

- Within-subjects correlations between each ROI's levels of activation and $\operatorname{WML}(3,4$, or 5 items) were calculated using nonparametric Spearman correlations.

- Between subjects differences for each ROI were assessed using one-tailed $t$-tests.

- For the longitudinal study, within-subjects changes over time for each ROI were assessed using paired $t$-tests.

\section{RESULTS}

The first part of this section describes the cross-sectional study (TBI group versus Control group); the second part describes the longitudinal study (TBI group at Time 1 versus Time 2).

\section{CROSS-SECTIONAL STUDY}

\section{Behavioral results: within-group analysis}

Spearman correlation coefficients indicated a decline in both accuracy and RT with increasing WML in the control group. A decline in accuracy with increasing WML was observed for the TBI group, but no such effects were noted for RT (Table 3). 


\section{Behavioral results: between-groups analysis}

Linear mixed model analyses for response accuracy indicated significant main effects of Group $(F=9.635, p=0.003)$ and WML $(F=17.356, p<0.001)$. There were no Group by WML interactions $(F=1.213, p=0.321)$. Post hoc analyses with LSD corrections using one-tailed statistics suggested statistically significant effects (all $p<0.10$ ) between all pairwise comparisons of WML. This suggests that our spatial working memory task is not only sensitive in detecting case/control differences but also shows the expected dose-response relationship between response accuracy and WML, with less accurate responding observed with increasing WML.

Similarly, linear mixed model analyses for RT indicated significant main effects of Group $(F=28.188, p<0.001)$ and WML $(F=2.767, p=0.052)$. There were no Group by WML interactions $(F=0.173, p=0.914)$. Post hoc analyses with LSD corrections using one-tailed statistics suggested statistically significant effects (all $p<0.10$ ) between the following pairwise comparisons

Table 3 | Within-groups performance effects.

\begin{tabular}{lll}
\hline & TBI group & Control group \\
\hline Correlation between & $r=-0.645 ;$ & $r=-0.843 ;$ \\
WML and accuracy & $p<0.001^{*}$ & $p<0.001^{*}$ \\
Correlation between & $r=-0.179 ;$ & $r=-0.403 ;$ \\
WML and response time & $p=0.244$ & $p=0.007^{*}$ \\
\hline
\end{tabular}

*Statistically significant result. of WML: 1 versus 4 and 5; 3 versus 4 and 5, indicating significant differences in RT from the baseline and easy conditions (load of 1 and 3) compared to the harder and more demanding conditions (load of 4 and 5). The Control group performed faster than the TBI group, regardless of WML. Also, across groups, participants were faster on the higher, and thus more difficult, WML trials (4 and 5) compared to the easier trials ( 1 and 3 ), with the longest time taken on the Baseline condition (WML of 1) in both groups. Results are summarized in Figure 2.

Post hoc analysis (see Table 4) show groups differences for accuracy and RTs in all conditions, with the exception of accuracy during the Baseline condition ( $\mathrm{WML}=1$ ). Both groups were indeed close to $100 \%$ accuracy on the Baseline condition (96 and 99\%, respectively for TBI and Controls).

\section{Imaging results and qualitative analysis: network recruited by the spatial working memory task}

Differences between groups were visible on statistical maps for each condition. In all cases, however, a common network of activated areas was observed in both groups, as follows: cingulate cortex (anterior and posterior); frontal cortex, including: frontal gyri (superior, middle, and inferior) and precentral gyrus; parietal cortex, including: supramarginal gyrus, angular gyrus, parietal lobule, and post-central gyrus; temporal cortex (medial and inferior temporal gyri); occipital cortex; subcortical structures, including: head of caudate nucleus, parahippocampal gyrus, putamen, thalamus and insula.

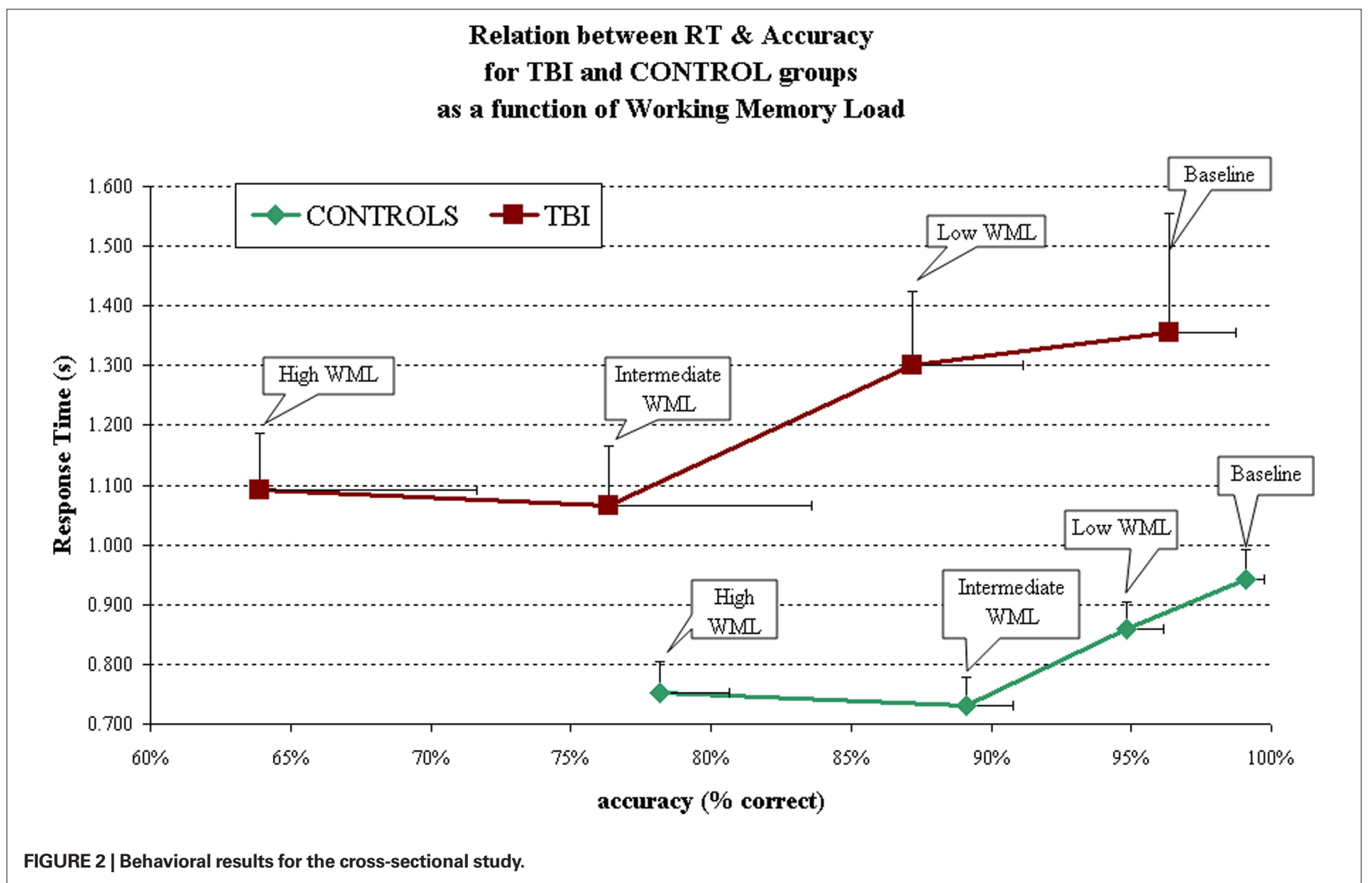




\section{Imaging results and quantitative analysis of regions of interest:} within-subjects effect of working memory load

Two ROI, ACC and ISMC, were defined as described in the section "Materials and Methods" and compared with regards for WML. Comparison of "Low WML" to "High WML" contrasts for the TBI

Table $4 \mid \boldsymbol{p}$ values forTBI and Control groups comparisons on the behavioral measures.

\begin{tabular}{lllll}
\hline & Baseline & LowWML & Intermediate WML & HighWML \\
\hline Accuracy & 0.139 & $0.040^{*}$ & 0.051 & $0.048^{*}$ \\
RT & $0.028^{*}$ & $0.002^{*}$ & $0.004^{*}$ & $0.003^{*}$
\end{tabular}

*Statistically significant result. group showed significant difference for the ACC $(p=0.049)$ but not for the ISMC ( $p=0.127)$. The opposite was observed in the Control group, where the comparison of "Low WML" to "High WML" showed no difference for the ACC ( $p=0.387)$ but a significant difference was observed for the ISMC $(p=0.005)$. Figure 3 illustrates these results.

In the TBI group, activity in the ACC was associated (as a trend) with WML $(r=0.327 ; p=0.06)$ but activity in ISMC was not $(r=-0.210 ; p=0.24)$. In the Control group, the opposite was observed: activity in the ACC was not correlated with WML $(r=0.052 ; p=0.76)$ but correlation of the activity in the ISMC was significant, albeit in a negative direction $(r=-0.462$; $p=0.005)$.

\section{ROI analysis: TBI group}

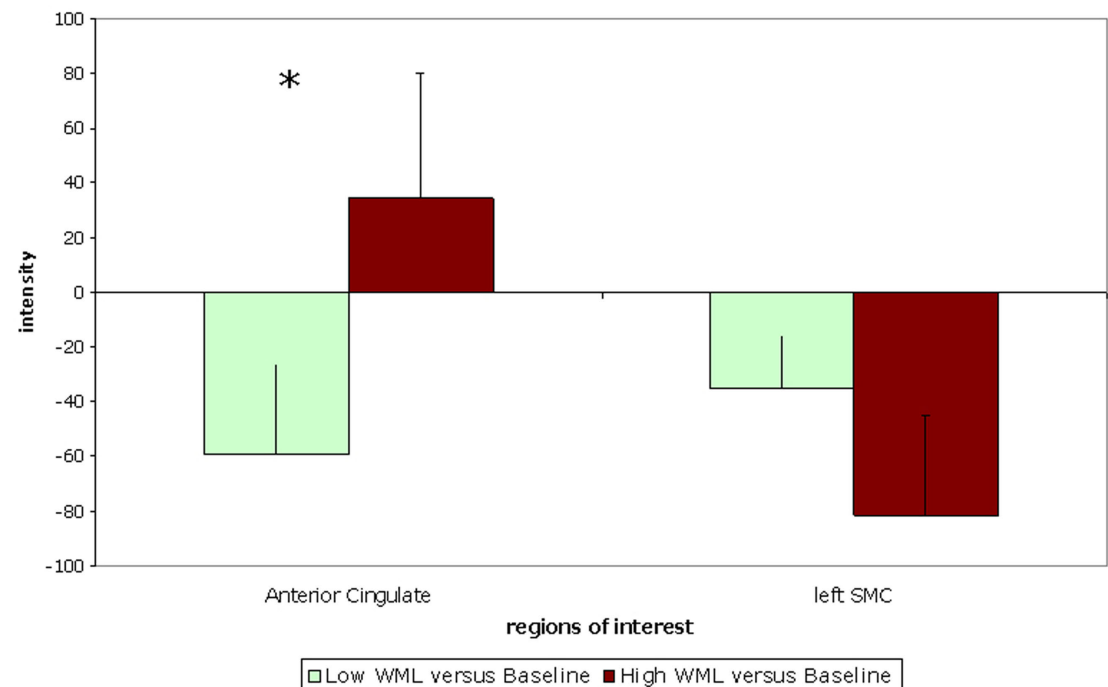

ROI analysis: Control group

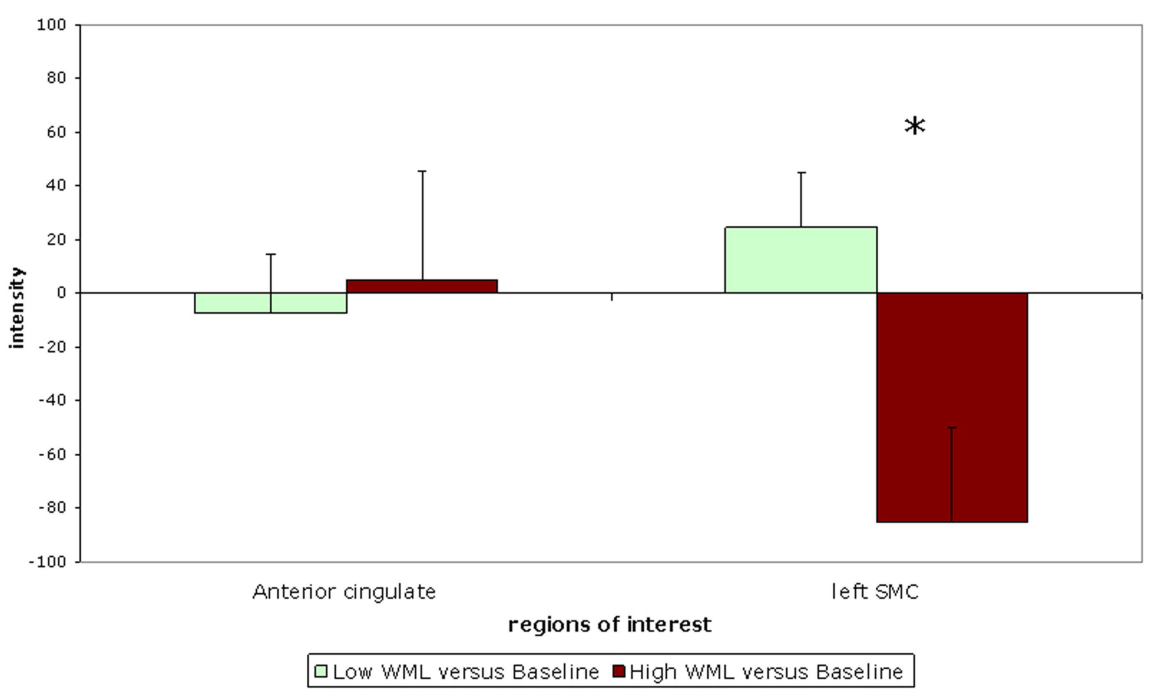

FIGURE 3 | Comparison of activation in regions of interest for "Low WML" (green) and "High WML" (brown) contrasts in traumatic brain injury group and Control group respectively. 


\section{Imaging results and quantitative analysis of regions of interest: between-subjects effect of working memory load}

The use of a parametric task allowed us to examine the effect of WML by analyzing the contrast "WML increase" (WML $=5$ items versus $\mathrm{WML}=3$ items). For the ACC region, a significant group difference was observed ( $p=0.038$ ), with the TBI group exhibiting more activation than the Control group. There was no observed group difference for this contrast, however, for the ISMC region $(p=0.356)$.

\section{LONGITUDINAL STUDY}

\section{Behavioral results}

As summarized in Table 5 and Figure 4, the TBI patients' performances were compared between Time 1 and Time 2 (approximately 12 months apart).

\section{Imaging results}

Activation was observed in the ACC and the head of caudate nucleus for the "Time 1 versus Time 2" contrast, while activation of ISMC was observed for the contrast "Time 2 versus Time 1," as shown in Figure 5.

\section{Table $5 \mid \boldsymbol{p}$ values forTime 1 versus Time 2 behavioral data for the} TBI patients.

\begin{tabular}{lll}
\hline & Accuracy & RT \\
\hline Baseline & 0.394 & 0.103 \\
LowWML & 0.117 & $0.047^{*}$ \\
Intermediate WML & 0.263 & $0.049^{*}$ \\
High WML & 0.289 & $0.032^{*}$
\end{tabular}

*Statistically significant result.
Between Time 1 and Time 2, the change of activation in ACC was not significant $(p=0.321)$ but the activation of ISMC was significantly increased at Time 2 as compared to Time $1(p=0.032)$.

\section{DISCUSSION}

\section{CROSS-SECTIONAL STUDY: PERFORMANCE}

Adolescent TBI patients showed worse behavioral performance (higher RTs and poorer accuracy) than Controls on the spatial working memory task. This finding is consistent with an extended literature of cognition after brain injury, which reports that pediatric patients with moderate to severe TBI experience psychomotor slowing and impaired working memory (see Babikian and Asarnow, 2009 for a review). It is important to note that the composition of the groups may have influence this result because gender was not equally distributed across the TBI and Control groups. Education level was not tested and difference of education level between groups cannot be excluded.

\section{CROSS-SECTIONAL STUDY: BRAIN IMAGING}

In both groups, the spatial working memory task activated a broadly distributed network involving the prefrontal cortex, the cingulate cortex, the pre and post-central gyri, wide areas of the parietal and occipital cortices, medial and inferior temporal gyri, as well as subcortical structures, notably the head of the caudate nucleus. These findings are consistent with literature of spatial working memory (Wager and Smith, 2003; D’Esposito, 2008). Quantitative differences between groups were observed, and this also is consistent with the literature on brain imaging of TBI (Hillary et al., 2002; Cazalis et al., 2006; Niogi et al., 2008).

Longitudinal TB I: RESP ONSE TIMES

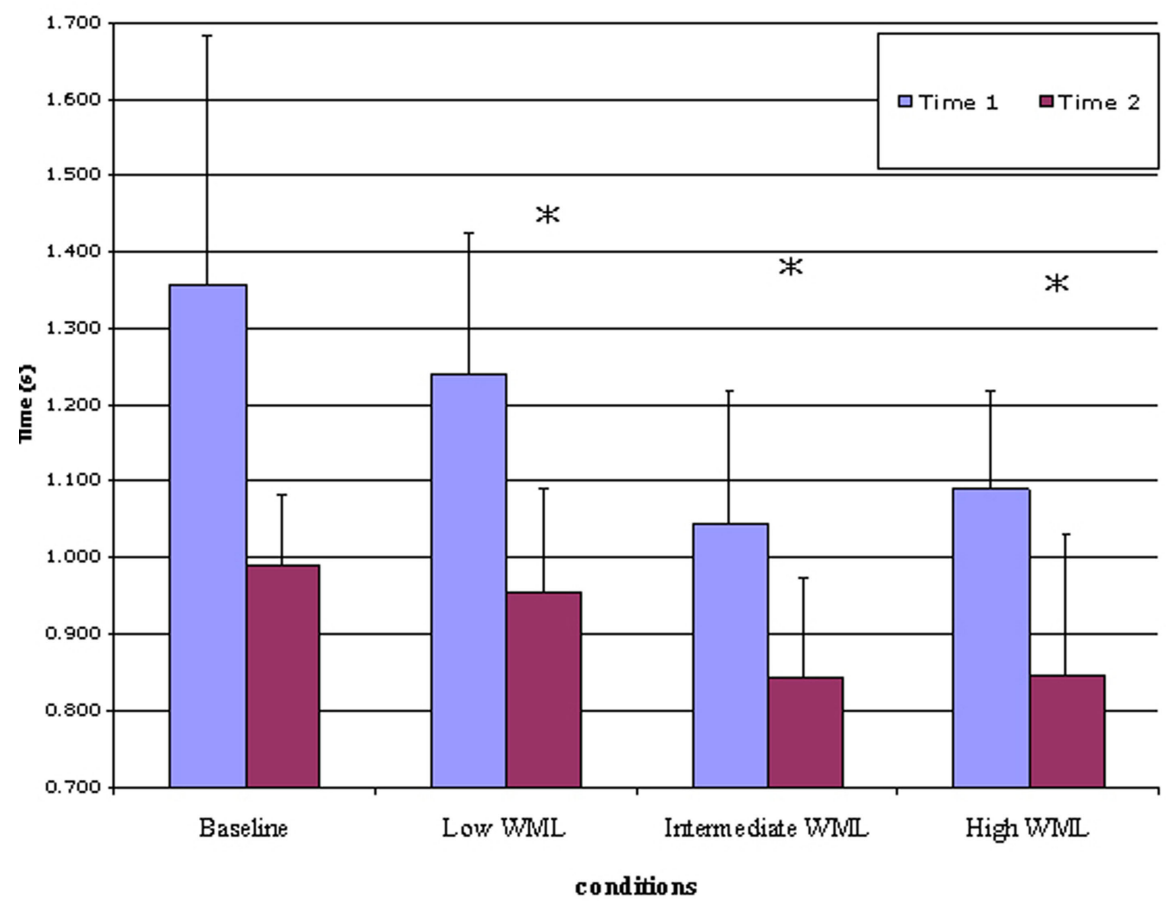

FIGURE 4 | Significant decrease of response times at Time 2 versus Time 1 in TBI patients. 

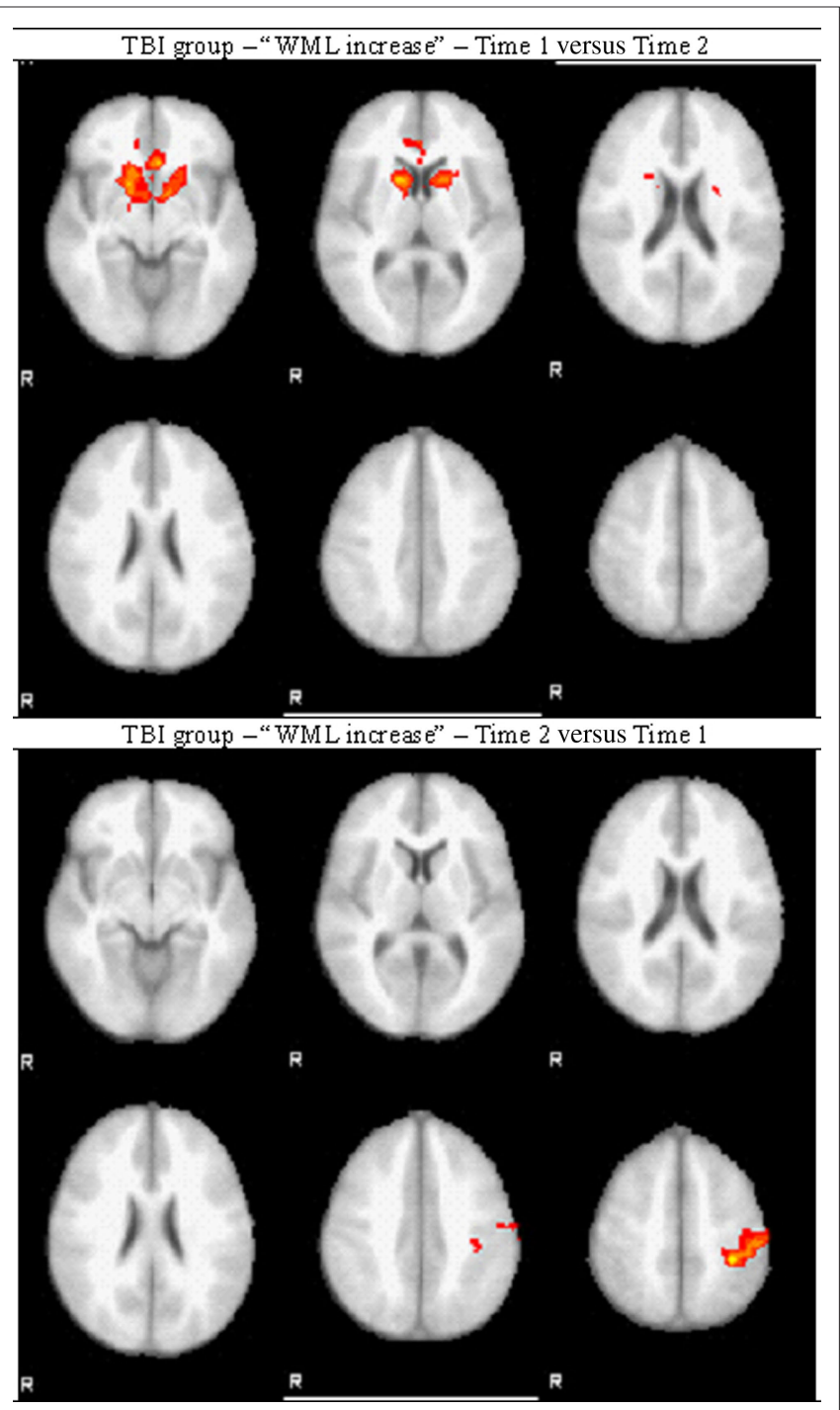

FIGURE 5 | Longitudinal contrasts forTBI patients.

The region of interest analysis was focused on the ACC, in order to understand its role in executive functions after moderate to severe TBI, and on the ISMC as well, because of its established role in spatial working memory.

Quantitative within-subject analyses of ROI activity in response to WML showed that, in the TBI group, ACC was significantly more activated when WML increased, while there was no change of ISMC activation. In the Control group, the opposite was observed: there was no change in ACC activation between conditions while ISMC activation decreased when WML increased. Our interpretation of those results is that Control subjects and TBI patients do not rely on recruiting the same cortical areas in response to increasing WML: Control subjects showed decreased activation of ISMC while TBI patients showed increased activation of ACC. Corroborating this result, correlation analysis showed that ACC activation was associated (as a trend) with WML in TBI patients but not in Controls, and that ISMC activation was correlated (inversely) in Controls but not in TBI patients. This double dissociation suggests that ACC activation depends on WML in TBI patients but not in Controls, while ISMC activation depends on WML in Controls but not in TBI patients.

Between-subject analysis, that compared TBI and Control groups for "WML increase," showed that ACC activation was significantly increased in the TBI group as compared to the Control group. In contrast, there was no difference between groups regarding ISMC activation. These results brought supplementary precisions to the findings above: ACC activation in TBI significantly increased in response to task difficulty as compared to Controls. Control subjects apparently responded to difficulty by decreasing activation of ISMC, while such decrease could not be observed in TBI patients who showed very low ISMC activation from the beginning.

Our interpretation of these results is that, although TBI patients and Control subjects seemed to use similar cognitive processes and neural networks in order to solve a spatial working memory task, there were notable differences between groups in the way these networks were recruited in response to difficulty, i.e., increasing WML in our case. Such differences, which were strongly suggested by the differences of performance during task solving, were revealed by comparing activation of two of the areas composing this network: ACC and ISMC.

While measure of clear differences between TBI and Control groups is an encouraging finding, we are aware that this may be only one part of a larger picture and that further research should be done with larger samples in order to determine the behavior of other areas involved in processing of items in spatial working memory, such as intraparietal cortex and dorsolateral prefrontal cortex.

\section{LONGITUDINAL STUDY: PERFORMANCE}

Six TBI patients were able to return for a second experimental session an average of 12 months after Time 1. While they did not show improvement in accuracy, their RTs were significantly decreased at Time 2. Recovery of cognitive functions after TBI has been extensively studied and our results are highly consistent with the existing literature. A recent meta-analysis of cognitive outcome after TBI (Babikian and Asarnow, 2009) showed that there is substantial recovery of processing speed in TBI patients over time, while recovery of working memory was noted in patients presenting with the most severe TBI.

\section{LONGITUDINAL STUDY: BRAIN IMAGING}

Within-subject analysis was a comparison of "WML increase" (WML of 5 items versus WML of 3 items) between Time 1 and Time 2. ACC and the head of caudate nucleus were more activated post-acutely as compared to the chronic phase, while ISMC was more activated in the chronic phase as compared to the post-acute phase; that is after a period of time allowing for recovery. These results, despite the small sample size, confirm our cross-sectional findings suggesting that prior to recovery, TBI patients significantly recruit ACC region for working memory, but they switch to ISMC recruitment following a period of recovery. Quantitative analyses of ROI activation in response to difficulty confirmed the increase of ISMC recruitment after recovery. This should be interpreted with regard to the cross-sectional study, which demonstrated a decreased recruitment of ISMC in response to difficulty (WML 
increase) in Controls while ISMC activation was consistently low in the TBI group. The fact that we observed more ISMC activation in TBI patients at Time 2 as compared to Time 1 suggests that they recovered the ability to use this area, although it is unclear if it follows the activation pattern observed in Controls. Moreover, it is important to note that because there was no longitudinal control group, we cannot exclude that such changes would not be due to recovery but simply to the fact that patients were one year older at Time 2, or may even have emerged by chance. This limitation, as well as the low number of subjects, makes the results of this longitudinal study difficult to interpret, but nevertheless encouraging, because they do show a change of activation pattern overtime, which parallels improvement in behavioral performance. It is reasonable to postulate that the changes of brain activation and behavioral performance observed in the TBI group between Time 1 and Time 2 result in the TBI group resembling the Control group over time, suggesting normalization and recovery.

\section{FUNCTIONS OF ANTERIOR CINGULATE CORTEX}

Over the last decade, several theories of ACC functions have been proposed, with some overlapping and some contradicting elements. Activation in ACC is observed in solving of difficult problem, multiple response management, conflict management, attention monitoring, and error detection (Botvinick et al., 2004; Ullsperger and Von Cramon, 2004; Declerck et al., 2006; Carter and van Veen, 2007; Hyafil et al., 2009; Ursu et al., 2009). In our study, only correct trials were included in brain imaging processing, therefore activation of the ACC cannot be attributed to error detection. In their study, Ursu et al. (2009) showed that there may be activation of ACC in presence of conflict even if the subject is not aware of such a conflict. Due to the structure of the task used in our study, it is not likely that the observed ACC activation was due to response override or conflict management. Magno et al. (2006) have indeed suggested that ACC activity is not related to error but to evaluation of difficulty. Furthermore, another study has shown that increased ACC activity was independent of anticipated error or conflict (Aarts et al., 2008). Finally, a study of TBI patients showed that ACC activity is increased with cognitive fatigue as experienced during effortful tasks (Kohl et al., 2009). This suggests that models of ACC functioning should include the broader concept of task difficulty - also described as challenging, effortful or demanding cognitive tasks -, thus encompassing concepts of error detection and conflict management.

Botvinick et al. (2004) have proposed an integrative hypothesis of ACC monitoring attention that applies to our results. According to their theory, activation of the ACC is associated with compensatory adjustment in response to tasks "experienced as cognitively difficult," considering that "conflict might serve as an index of the demand for mental effort," but that difficulty can occur in absence of conflict. While TBI subjects in our study did not perform as well as Controls despite exhibiting the highest ACC activation, this is not sufficient to exclude a compensatory adjustment. The compensatory adjustment theory, however, is used to explain studies showing that ACC activation is associated with higher performance (Botvinick et al., 2001; Garavan et al., 2002; Kerns et al., 2004). The results of our study apparently contradict those results, since TBI patients exhibited worse performance than Controls. However, in a previous fMRI study, we have shown that adult patients with severe TBI who perform very poorly on a planning task with working memory components exhibited reduced activation of the ACC as compared to severe TBI patients who were able to perform as well as Control subjects (Cazalis et al., 2006). Similar results were described by Kohl et al. (2009) as a coping mechanism. It is therefore reasonable to assume that those TBI patients in our current study, who were able to complete the task, may have recruited ACC in a compensatory adjustment fashion, in order to avoid poorer performance.

Alternatively, other studies of individual performance and cortical activation variability suggest that activity in ACC could reflect the ability of the individual to succeed at the task, in which case ACC activation could be seen as a predictor of performance. In a review of PET studies, Paus et al. (1998) argued that ACC activation positively reflects the level of difficulty of a task, which is itself indicative of the level of performance. In a study of a planning task, Cazalis et al. (2003) showed that subjects with poorer performance exhibited greater activation of the ACC than subjects with better performance. Hahn et al. (2007) have shown that ACC activity is dependent on performance (RTs). In two studies of verbal working memory, Osaka et al. $(2003,2004)$ found greater ACC activation in individuals with higher verbal working memory spans. Although it is difficult to transpose verbal WM and spatial WM, these studies interestingly confirm the dependence of ACC activation on performance. Furthermore, another study of anatomical variation of ACC demonstrated an association between a specific (leftward) morphological pattern of paracingulate sulcus folding and higher performance in demanding cognitive tasks (Fornito et al., 2004).

Such accumulation of results, including conflicting data, may lead the reader to a broad vision of ACC as a backup system that takes over problem solving when the executive system is overloaded. In simple words, ACC could be the ace up the prefrontal cortex's sleeve, meant to be used when executive functions are impaired or overloaded, whether by physical disconnection as in TBI or simply by a high level of difficulty. As a backup system, its efficiency would be limited, but would be involved in improving performance up to a certain limit, and would remain activated after this limit has been reached. This hypothesis is compatible with the concept of compensatory adjustment and would explain why some studies show that individuals with ACC lesions are still able to solve difficult problems (di Pellegrino et al., 2007). Furthermore, as suggested by our results, a double dissociation between ACC and ISMC activation in response to difficulty could account for the conflicting literature regarding the role of ACC.

\section{CONCLUSION}

While our study provides yet another small brick to the construction of ACC understanding, it is noteworthy that the ACC is involved in so many apparently distinct functions that one may have to reconsider the existing theoretical framework before one can reach firm conclusions. One aspect that deserves further consideration is the structure-function relationship that exists at the level of the ACC (Koski and Paus, 2000; Margulies et al., 2007; Orr and Weissman, 2009) as it exists in other regions. For example, one appealing model has been proposed that connects the 
structure of dorsolateral prefrontal cortex to its functions, with concrete action planning being closer to motor areas and more abstract planning being more rostral (Koechlin and Jubault, 2006; Badre and D'Esposito, 2007), consistently with the perceptionaction model that was developed by Fuster (2005). A similarly innovative proposition was made by Postle (2006), stating that working memory is an emergent function rather than an embedded function, a theory also supported by Zimmer (2008). The ACC occupies a strategic location in the brain and therefore has the capacity of integrating of a wide variety of inputs. Indeed, ACC activity has been associated with a wide variety of mental functions, such as attention (Brocki et al., 2009), motivation (Ullsperger and Von Cramon, 2004), sexuality (Stoléru et al., 1999), pain (Robinson et al., 2009), executive functions (Mansouri et al., 2009), among others.

Most models, deeply influenced by Cartesian heritage, draw a scientific line between emotional and intellectual processes, despite our knowledge that these functions are highly interdependent. In order to fully understand how one cerebral area can process such diverse

\section{REFERENCES}

Aarts, E., Roelofs, A., and van Turennout, M. (2008). Anticipatory activity in anterior cingulate cortex can be independent of conflict and error likelihood. J. Neurosci. 28, 4671-4678.

Allman, J. M., Hakeem, A., Erwin, J. M., Nimchinsky, E., and Hof, P. (2001). The anterior cingulate cortex: the evolution of an interface between emotion and cognition. Ann. N. Y. Acad. Sci. 935, 107-117.

Babikian, T., and Asarnow, R. (2009). Neurocognitive outcomes and recovery after pediatric TBI: metaanalytic review of the literature. Neuropsychology 23, 283-296.

Badre, D., and D'Esposito, M. (2007). FMRI evidence for a hierarchical organization of the prefrontal cortex. J. Cogn. Neurosci. 19, 1-18.

Botvinick, M. M., Braver, T. S., Barch, D. M., Carter, C. S., and Cohen, J. D. (2001). Conflict monitoring and cognitive control. Psychol. Rev. 108, 624-652.

Botvinick, M. M., Cohen, J. D., and Carter, C. S. (2004). Conflict monitoring and anterior cingulate cortex: an update. Trends in Cogn. Sci. 8, 539-546.

Brocki, K., Clerkin, S. M., Guise, K. G., Fan, J., and Fossella, J. A. (2009). Assessing the molecular genetics of the development of executive attention in children: focus on genetic pathways related to the anterior cingulate cortex and dopamine. Neuroscience 164, 241-246.

Carter, C. S., and van Veen, V. (2007). Anterior cingulate cortex and conflict detection: an update of theory and data. Cogn. Affect. Behav. Neurosci. 7, 367-379.
Cazalis, F., Feydy, A., Valabregue, R., Pelegrini-Issac, M., Pierot, L., and Azouvi, P. (2006). fMRI study of problem-solving after severe traumatic brain injury. Brain Inj. 20, 1019-1028.

Cazalis, F., Valabregue, R., Pelegrini-Issac, M., Asloun, S., Robbins, T. W., and Granon, S. (2003). Individual differences in prefrontal cortical activation on the Tower of London planning task: implication for effortful processing. Eur. J. Neurosci. 17, 2219-2225.

Declerck, C. H., Boone, C., and De Brabander, B. (2006). On feeling in control: a biological theory for individual differences in control perception. Brain Cogn. 62, 143-176.

D’Esposito, M. (2008). "Working memory," in Handbook of Clinical Neurology, eds P. J. Vinken and G. W. Bruyn (Amsterdam: Elsevier), 237-247.

di Pellegrino, G., Ciaramelli, E., and Ladavas, E. (2007). The regulation of cognitive control following rostral anterior cingulate cortex lesion in humans. J. Cogn. Neurosci. 19, 275-286.

Fiehler, K., Burke, M., Engel, A., Bien, S., and Rösler, F. (2008). Kinesthetic Working Memory and Action Control within the Dorsal Stream. Cereb. Cortex 18, 243-253.

Fornito, A., Yucel, M., Wood, S., Stuart, G. W., Buchanan, J. A., Proffitt, T., Anderson, V., Velakoulis, D., and Pantelis, C. (2004). Individual differences in anterior cingulate/paracingulate morphology are related to executive functions in healthy males. Cereb. Cortex 14, 424-431.

Fuster, J. M. (2005). Cortex and Mind: Unifying Cognition. New York: Oxford University Press.

information, we may have to rethink those categories and investigate their potential interconnections. A neurophenomenological approach would suggest that all inputs processed by one area have to share either a common architecture or require a common processing (Allman et al., 2001). Therefore, seeking the common ground of these inputs would provide essential information on the fundamental roles of the ACC. It is likely that such an integrative theory would help unifying the current models of ACC functions.

\section{ACKNOWLEDGMENTS}

We want to thank Bernard Mendiburu for Matlab programming and support, Russell Poldrack for help with study design, Trent Thixton for help with brain imaging, Tarman Aziz for proofreading, Claudia Kernan and Nina Newman for patients' assessment and consenting, all the RAPBI members for discussion and feedback. This work was supported by the UCLA Brain Injury Research Center (Christopher Giza, Robert F. Asarnow), The Della Martin Foundation, Winokur Family Foundation/Child Neurology Foundation (Christopher Giza), UCLA Faculty Grants Program.

Garavan, H., Ross, T. J., Murphy, K. Roche, R.A. P., and Stein, E. A. (2002). Dissociable executive functions in the dynamic control of behavior: inhibition, error detection, and correction Neuroimage 17, 1820-1829.

Ghajar, J., and Ivry, R. B. (2008). The predictive brain state: timing deficiency in traumatic brain injury? Neurorehabil. Neural. Repair 22, 217-227.

Hahn, B., Ross, T. J., and Stein, E. A. (2007). Cingulate activation increases dynamically with response speed under stimulus unpredictability. Cereb. Cortex 17 , 1664-1671.

Hanakawa, T., Dimyan, M. A., and Hallett, M. (2008). Motor planning, imagery, and execution in the distributed motor network: a time-course study with functional MRI. Cereb. Cortex. 18, 2775-2788.

Hillary, F., Steffener, J., Biswal, B., Lange, G., DeLuca, J., and Ashburner, J. (2002). Functional magnetic resonance imaging technology and traumatic brain injury rehabilitation: guidelines for methodological and conceptual pitfalls. J. Head Trauma Rehabil. 17, 411-430.

Hyafil, A., Summerfield, C., and Koechlin, E. (2009). Two mechanisms for task switching in the prefrontal cortex. J. Neurosci. 29, 5135-5142.

Kerns, J. G., Cohen, J. D., MacDonald, A. W. III, Cho, R. Y., Stenger, V. A., and Carter, C.S. (2004). Anterior cingulate conflict monitoring and adjustments in control. Science 303, 1023-1026.

Koechlin, E., and Jubault, T. (2006). Broca's area and the hierarchical organization of human behavior. Neuron 50, 963-974.

Kohl, A. D., Wylie, G. R., Genova, H. M., Hillary, F.G., and DeLuca,J. (2009). The neural correlates of cognitive fatigue in traumatic brain injury using functional MRI. Brain Inj. 2, 420-432.

Koski, L., and Paus, T. (2000). Functional connectivity of the anterior cingulate cortex within the human frontal lobe: a brain-mapping meta-analysis. Exp. Brain Res. 133, 55-65.

Magno, E., Foxe, J. J., Molholm, S., Robertson, I. H., and Garavan, H. (2006). The anterior cingulate and error avoidance. J. Neurosci. 26, 4769-4773.

Mai, J. K., Paxinos, G., and Assheuer, J. K. (2004). Atlas of the Human Brain, 2nd Edn. San Diego, CA: Academic Press.

Mansouri, F. A., Tanaka, K., and Buckley, M. J. (2009). Conflict-induced behavioural adjustment: a clue to the executive functions of the prefrontal cortex. Nat. Rev. Neurosci. 10, 141-152.

Margulies, D. S., Kelly, A. M., Uddin, L. Q., Biswal, B. B., Castellanos, F. X., and Milham, M. P. (2007). Mapping the functional connectivity of anterior cingulate cortex. Neuroimage 37, 579-588.

Metting, Z., Rödiger, L. A., De Keyser, J., and van der Naalt, J. (2007). Structural and functional neuroimaging in mildto-moderate head injury. Lancet Neurol. 6, 699-710.

Niogi, S. N., Mukherjee, P., Ghajar, J., Johnson, C. E., Kolster, R., Lee, H., Suh, M., Zimmerman, R. D., Manley, G. T., and McCandliss, B. D. (2008) Structural dissociation of attentional control and memory in adults with and without mild traumatic brain injury. Brain 131, 3209-3221.

Orr, J. M., and Weissman, D. H. (2009). Anterior cingulate cortex makes 2 contributions to minimizing distraction. Cereb. Cortex 19, 703-711. 
Osaka, M., Osaka, N., Kondo, H., Morishita, M., Fukuyama, H., Aso, T., and Shibasaki, H. (2003). The neural basis of individual differences in working memory capacity: an fMRI study. Neuroimage 18, 789-797.

Osaka, N., Osaka, M., Kondo, H., Morishita, M., Fukuyama, H., and Shibasaki, H. (2004). The neural basis of executive function in working memory: an fMRI study based on individual differences. Neuroimage 21, 623-631.

Paus, T., Koski, L., Caramanos, Z., and Westbury, C. (1998). Regional differences in the effects of task difficulty and motor output on blood flow response in the human anterior cingulate cortex: a review of 107 PET activation studies. Neuroreport 9, R37-R47.

Pepin Press. (2006a). The Agile Rabbit Visual Dictionary of Vegetables, Agile Rabbit Editions. Amsterdam: Pepin Press.

Pepin Press. (2006b). The Agile Rabbit Visual Dictionary of Fruits, Agile Rabbit Editions. Amsterdam: Pepin Press.

Postle, B. R. (2006). Working memory as an emergent property of the mind and brain. Neuroscience 139, 23-38.

Rasmussen, I.-A., Xu, J., Antonsen, I. K., Brunner, J., Skandsen, T., Axelson, D. E., Berntsen, E. M., Lydersen, S., and
Håberg, A. (2008). Simple dual tasking recruits prefrontal cortices in chronic severe traumatic brain injury patients, but not in controls. J. Neurotrauma 25, 1057-1070.

Ratiu, P., and Talos, I.-F. (2006). CrossSectional Atlas of the Brain. Cambridge, MA: Cambridge Harvard University Press.

Robinson, M., Edwards, S., Iyengar, S., Bymaster, F., Clark, M., and Katon, W. (2009). Depression and pain. Front. Biosci. 1, 5031-5051.

Sack, A. T., Jacobs, C., Martino, F. D., Staeren, N., Goebel, R., and Formisano, E. (2008). Dynamic premotor-to-parietal interactions during spatial imagery. J. Neurosci. 28, 8417-8429.

Sanchez-Carrion, R., Fernandez-Espejo, D., Junque, C., Falcon, C., Bargallo, N., Roig, T., Bernabeu, M., Tormos, J. M., and Vendrell, P. (2008). A longitudinal fMRI study of working memory in severe TBI patients with diffuse axonal injury. Neuroimage 43, 421-429.

Shaw, M. E., Moores, K. A., Clark, R. C., McFarlane, A. C., Strother, S. C., Bryant, R.A., Brown, G. C., and Taylor, J. D. (2009). Functional connectivity reveals inefficient working memory systems in post-traumatic stress disorder. Psychiatry Res. 172, 235-241.

Smith, S. M., Jenkinson, M., Woolrich, M. W., Beckmann, C. F., Behrens, T.
E. J., Johansen-Berg, H., Bannister, P. R., De Luca, M., Drobnjak, I., Flitney, D. E., Niazy, R. K., Saunders, J., Vickers, J., Zhang, Y., De Stefano, N., Brady, J. M., and Matthews, P. M. (2004). Advances in functional and structural MR image analysis and implementation as FSL. Neuroimage 23(Suppl. 1), S208-S219.

Stoléru, S., Grégoire, M., Gérard, D., Decety, J., Lafarge, E., Cinotti, L., Lavenne, F., Le Bars, D., Vernet-Maury, E., Rada, H., Collet, C., Mazoyer, B., Forest, M. G., Magnin, F., Spira, A., and Comar, D. (1999). Neuroanatomical correlates of visually evoked sexual arousal in human males. Arch. Sex. Behav. 28, 1-21.

Ullsperger, M., and Von Cramon, D. Y. (2004). Neuroimaging of performance monitoring: error detection and beyond. Cortex 40, 593-604.

Ursu, S., Clark, K. A., Aizenstein, H. J., Stenger, V.A., and Carter, C. S. (2009). Conflict-related activity in the caudal anterior cingulate cortex in the absence of awareness. Biol. Psychol. 80, 279-286.

Wager, T. D., and Smith, E. E. (2003). Neuroimaging studies of working memory: a meta-analysis. Cogn. Affect. Behav. Neurosci. 3, 255-274.

Woolrich, M., Jbabd, S., Patenaude, B., Chappell, M., Makni, S., Behrens, T., Beckmann, C., Jenkinson, M., and Smith, S. M. (2009). Bayesian analy- sis of neuroimaging data in FSL. Neuroimage 45(Suppl.), S173-S186.

Zimmer, H. D. (2008). Visual and spatial working memory: from boxes to networks. Neurosci. Biobehav. Rev. 32, 1373-1395.

Conflict of Interest Statement: The authors declare that the research was conducted in the absence of any commercial or financial relationships that could be construed as a potential conflict of interest.

Received: 05 October 2010; paper pending published: 05 November 2010; accepted: 16 December 2010; published online: 21 January 2011.

Citation: Cazalis F, Babikian T, Giza C, Copeland S, Hovda D and Asarnow RF (2011) Pivotal role of anterior cingulate cortex in working memory after traumatic brain injury in youth. Front. Neur. 1:158. doi: 10.3389/fneur.2010.00158

This article was submitted to Frontiers in Neurotrauma, a specialty of Frontiers in Neurology.

Copyright (c) 2011 Cazalis, Babikian, Giza, Copeland, Hovda and Asarnow. This is an open-access article subject to an exclusive license agreement between the authors and the Frontiers Research Foundation, which permits unrestricted use, distribution, and reproduction in any medium, provided the original authors and source are credited. 